\title{
Review of semi-active suspension based on Magneto-rheological damper
}

\author{
Musabyimana Josee $^{1 *}$, Kazima Sosthene ${ }^{2}$, Turabimana Pacifique ${ }^{2}$
}

${ }^{1}$ Electrical and Electronic Engineering Department, College science and Technology, University of Rwanda, 3900, Kigali, Rwanda

${ }_{2}^{2}$ Mechanical Engineering Department, Rwanda Polytechnic- IPRC Gishari, 60 Rwamagana, Rwanda

\begin{abstract}
Vehicle suspension system plays a critical role in transferring static and dynamic loads produced by the vibration of vehicle body
and wheels and absorbing shock vibration caused by road roughness. Low damping provides a better vehicle mass isolation and
it give a ride comfort and hard damping provides vehicle stability with good road holding. The need to enhance conflicting odds
between ride comfort and vehicle stability makes the design of the suspension a significant part particularly for off- road vehicles.
Passive suspension can't mitigate tradeoff between ride comfort and vehicle stability, because it presents a high frequency vibra-
tion. It is in this line various types of suspension, like semi- active suspension, active suspension and intelligent suspension have
been developed to reduce this compromise need. This paper aims to describe in details different types of vehicle suspensions,
their characteristics, and their working principles mode. It illustrates in details the magneto rheological fluid (intelligent fluid)
properties, compositions, mechanical model of Magneto-rheological damper like Bingham model and Bouc-Wen model. It also
reviews semi active suspension control strategies based on Magneto rheological damper, like skyhook, ground hook, sliding
mode, fuzzy logic and linear quadratic Gaussian. Simulations shows that a combination of more than two control strategies pro-
vide a better vehicle comfort and vehicle stability at the same time.
\end{abstract}
Keywords: Semi-active suspension, Magneto-rheological damper, control strategies,

\begin{tabular}{cll}
\hline History & Author Contacts & http://dx.doi.org/10.29228/eng.pers.50853 \\
Received: 13.04 .2021 & *Corresponding Author & \\
Accepted: 25.05.2021 & e-mail addresses: josee.musabyimana@gmail.com, kazima10@gmail.com \\
& Orcid numbers : :0000-0002-4427-130X & \\
\hline
\end{tabular}

\section{Introduction}

The main key responsibilities suspension is to ensure ride comfort and road holding. It transfers different load and ground forces to the vehicle body. A tradition vehicle suspension constitutes by a spring coil spring or air spring and a damping element. The coefficients of damping element and spring are selected based on comfort, road holding, and handling characteristics[1]. Even if conventional suspensions may thrive a trade-off between ride comfort and road holding as though their spring and damping coefficients cannot be adjustably tuned depending on driving efforts and road situation. It means that good ride comfort and road holding is gotten only under the designed circumstances. The studies and investigations on intelligent suspension with the development of microprocessor, sensors and actuators in 1980s have showed a great impact on avoiding the trade off by controlling the damping characteristics of suspensions. Based on the mode of generation of the desired control forces, intelligent suspension systems can be categorized into two main types: active and semi-active. The active suspension often requires significant amount of external energy to produce the desired control damping forces. It is achieved by using pneumatic or hydraulic actuators .

\subsection{Types of suspension}

In automotive dynamic era, suspension system is classified into primary and secondary, the primary suspension ensures the connection between vehicle body and wheels and secondary type represent the seat. There are two basic types of elements in tradition suspension systems. These elements are springs and dampers. The spring in a vehicle's suspension system ensure the mount of static weight of the vehicle and storage of energy applied to the vehicle body. The damper ensures the dissipation vibration energy and monitor different forces from the road transmitted to the vehicle. Basically the role and form of a suspension remain the same regardless of the category of suspension or automobile. Primary suspensions will be classified into passive, active and semi-active suspension.

\subsubsection{Passive suspension}

As it shown in the Figure 1 passive suspension is a type of suspension which implies the control of inherent odds between minimum-frequency and maximum-frequency vibration mitigation. 
This kind suspension system has of two important elements an energy evacuating element (shock absorber), which is the damper, and an energy-storing element, which is the spring. As these two elements can't give energy to the system, reason why this type of suspension systems is named passive. Passive suspension systems are exposed to different tradeoffs when they applied to a huge vibration frequencies[2]. Passive dampers figure out the conventional shock absorbers mostly applicable to many vehicles. They have the fixed damping characteristics, i.e. their damping characteristics cannot be adjusted while the car is being driven and thus, they are designed to achieve the most convenient trade-off between ride, road holding, and stability[3].

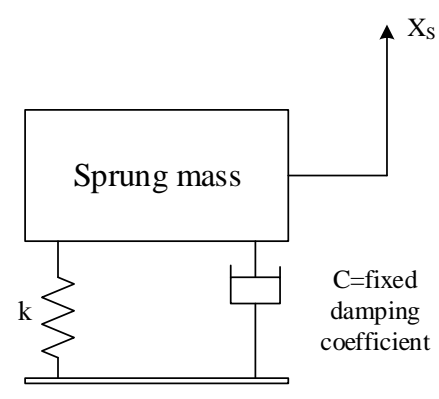

Figure 1 Passive suspension

A high damping suspension will lead best vehicle road handling. However, it transmits much of the road forces to the vehicle body. Whenever the vehicle is riding at low speed on a harsh road or at high speed in a straight line, this will be perceived as a tough ride. The harsh ride is obnoxious for the drivers because it can damage the payloads or cause an accident. A soft damping suspension will lead a more comfortable drive, but may substantially mitigates the stability while the car turning, changing the lane, or exiting the ramp. Best design of a passive suspension can adjust some extensions on improving ride and road handling, but cannot remove this odd as indicated in Figure 2[28].

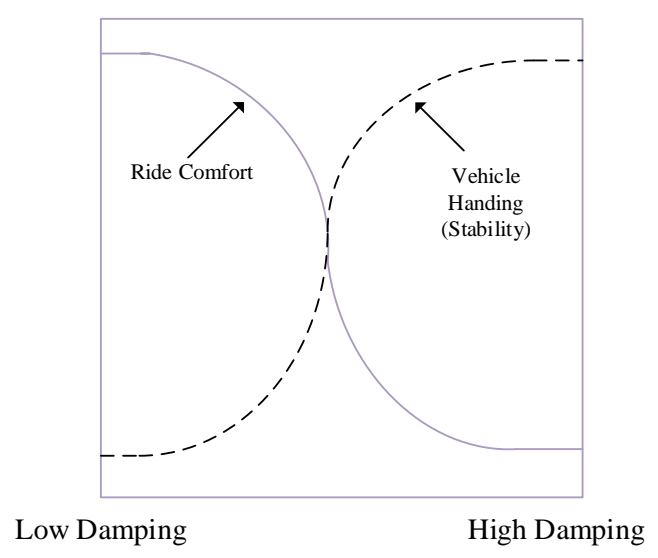

Figure 2 Passive suspension trade off

An ideal suspension system should provide good vibration isolation, i.e. minimum acceleration of the body mass, and ensure the maximal acceptable relative displacement between the vehicle body and different suspension components.

\subsubsection{Active suspension}

The active suspension is a kind of suspension where the passive shock absorber (damper) or both the passive damper and spring are replaced by an actuator force as it shown in the Figure 3. Actuator force has the capacity to the spring and the damper to add and dissipate energy from the system, inversely to a passive damper, can only dissipates energy. In an active suspension, necessary displacement and velocity is applied independently by the actuator force. In active suspension the active force is provided by actuator, which is monitored and controlled by a control algorithm utilizing data from sensors mounted to the vehicle[4]. The active suspension has two important parties' actuator and spring. The spring has a role supporting the static load of sprung mass and force actuator provides the necessary reactive force to mitigate or assimilate deflection caused by road abnormal. The actuator may be hydraulically, electromagnetically, pneumatically, or hybrid systems. These actuator systems are controlled and monitored by electric drives, the electric power of these drives is provided by the battery of vehicle or any other system in vehicle. Based to the high force ability, the easy design, advanced of technology, and commercial availability of the many parts, the hydraulic systems are commonly utilized in control of sprung systems of vehicle. The active suspension system equipped with electromagnetic actuator is named electromagnetic active suspension system; it is constituted by spring and electromagnetic actuator fixed in parallel combination between vehicle body and unsprung mass. The electromagnetic actuator is controlled by embedded systems which supply the required electric power of the actuator. It gives active controlled force to mitigate road shocks in small time, eliminates the roll and pitch vibration, and optimize road handling and ride comfort. When the actuator is operated and mounted mechanically it provides a high bandwidth active suspension and control body mass and unsprung mass. Low-bandwidth is provided to active suspension monitoring the vehicle body (sprung mass) when the active actuator operates and mounted mechanically in series with the spring and the damper. Generally, the range frequencies of the unsprung mass vary between $10-15 \mathrm{~Hz}$, and the range of sprung mass frequency vary between 1-2 Hz. The desired frequency bandwidth determines the price of active suspension. When the desired bandwidth is limited the cost of control force actuator is minimize as cost of active suspension is minimized also[5]. With high damping force of active suspension, the best ride control is gotten. Active suspension operates by constantly sensing various vibrations in the road surface and provides those signals to the ECU, to activate actuator components. Actuators react upon the system to change its behavior, adjusting shock absorber, spring rate and the like, to optimize ride comfort, drivability, and responsiveness. The ECU ensure the collections, analyzes and interpretations of data provided by sensors in little time like 10 milliseconds. Nowadays a new kind of active suspension are being developed with linear electromagnetic motors monitored and controlled by microprocessors and complex mathematics equations[6]. 


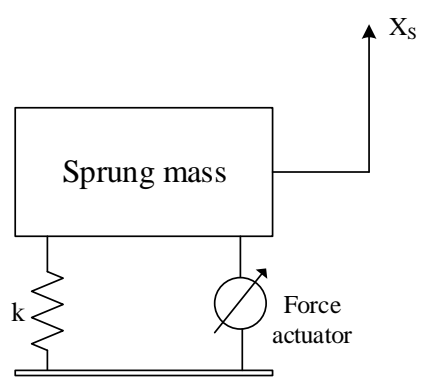

Figure 3 Active suspension

\subsubsection{Semi- active suspension}

Semi-active suspension systems have been investigated and studied in the early 1970's, the tradition spring element is remained in this kind of suspension, however the shock absorber damper is replaced with an adjustable and controllable damper as it is shown in Figure 4 below.

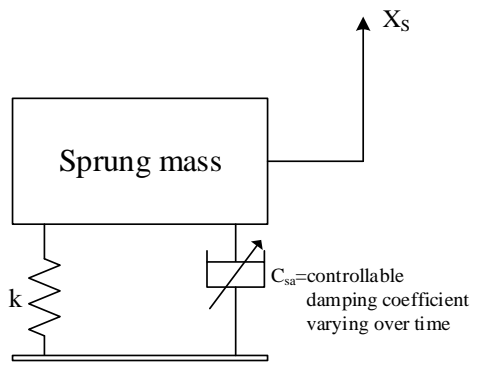

Figure 4. Semi-active suspension

Meanwhile an active suspension system requires an external energy source to activate an actuator which is controls and monitoring the vehicle. The semi-active system utilizes just external power sensors and microcontroller to change the damping characteristics of shock absorber. The embedded microcontroller provides the level of damping force based on a control strategy program to change the shock absorber characteristics to achieve that the necessary damping force. The semi-active suspension dampers adjust their damping force in real time according to a controller strategy which is often based on the system dynamics. By adjusting the damper's fluid resistance movement, or damping coefficient, changes the damper force. Based on its low cost and the capacity to change the damping coefficient independently of damper velocity, within limits, has increased a number of institutions companies and industries to investigate the ability of optimization suspension performance by utilizing semi-active damper technology[7].The implementation of semi-active control law, is based on real time adjustment of damping force. Nowadays, many semi-active dampers are using hydraulic or electromagnetic systems. The first type of semi-active utilizes mechanical valves which are activated by coil or stepper motor to control damper force in a hydraulic damper. The last kind of system uses controllable fluids like electro-rheological or magneto-rheological to change their rheological effect in order to provide the required damping force. Even if the cost of semi-active suspensions remains high compare to the passive suspensions, they are very cheaper compare to active suspensions; as such, they are considered as a more commercially viable option for many vehicles cat- egories including commercial vehicle. Many researches and studies have shown that semi-active presents the best performance of vehicle dynamic.

\section{Magneto rheological fluid}

Magneto-rheological (MR) materials are classified in smart materials, they are presently being applied many applications. Smart materials are the materials which physical properties can be adjusted or changes by external physical properties such as, temperature, $\mathrm{pH}$, moisture, stress, electric or magnetic fields. Magnetorheological material has various form of fluids; it can be a gel or even a solid material like elastomers. A magneto-rheological fluid mainly composed by micron-sized iron element that is immerged in carrier oil. Magneto rheological fluid presents the capacity of varying from free-moving liquid state into a semi-solid condition with limited fluid movement in fast response within some milliseconds when applied to the external magnetic field. During the middle of the 20th century, researchers and scientists started to work with fluids able of experiment physical changes in a little time approximatively 10 milliseconds, for example, rheological fluids change viscosity or became semi-solids when are subjected to magnetic fields or electric currents. As it said above their viscosity varies in little time which is less than ten milliseconds. In four decades ago, magneto-rheological fluids were introduced in automotive applications. The main effort were emphasized on autonomous suspensions from the first intelligent approaches[8]. In 1940s Jacob Rabinow [9] invented the fluid, which yield stress and viscosity changes when magnetic field is applied on it; since its invention different applications using MR fluid have been investigated and designed such as dampers, clutches, engine mounts, haptic devices and building dampers, etc[10]. Physical characteristics changes of MR fluid are resulted from the chain-like structures between paramagnetic MR particles in the low permeability solvent[11]. At the normal state, MR fluid presents the isotropic Newtonian behavior because the MR particles move freely as shown in Figure 5 (a). However, when the magnetic field is subjected to the MR fluid, MR fluid forms a chain structure as it is indicated in Figure 5 (b), MR fluids present the anisotropic Bingham behavior which result on resisting on flow and external shear stress. From this property, force or torque of application devices can be easily monitored by the density of magnetic field[12].
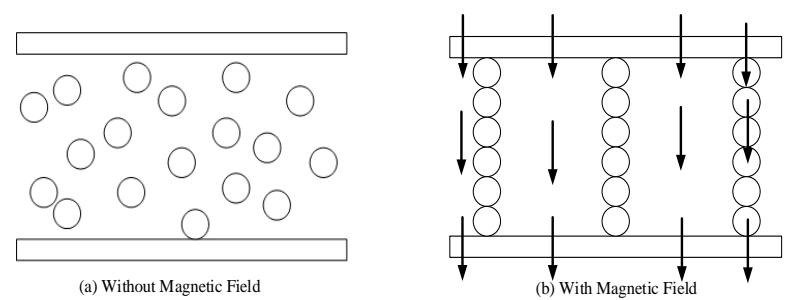

Figure 5 Magneto-rheological fluid

\subsection{Rheological properties of magneto-rheological fluid}

Rheology is the field which study and investigate the flow and changes of materials under exposed forces which is often measured by a rheometer. The process of measurement and test of rheological properties is applicable to all materials. Rheological 
properties can be measured from bulk sample twist utilizing a mechanical rheometer or on a micro-scale by using a micro capillary viscometer or an optical technique such as micro-rheology. Various mostly utilized materials and formulations show complex rheological properties, whose viscosity and visco-elasticity can change according to the external conditions subjected, such as temperature, $\mathrm{pH}$, moisture, stress, electric or magnetic fields. Rheological properties is also measured in medical application where it given by internal changes of biopharmaceutical element such as protein concentration, stability or any kind interaction of between its properties[13]. Characteristics effects of magneto rheological fluid is based on increasing or decrease the viscosity of a magnetorheological fluid and presenting yield stress based on a magnetic field applied on it as indicated by chain form of iron micro sized particles[14]. The MR effect is controlled and monitored by magnetic field intensity and rheological characteristics of magnetorheological fluid components. It means that rheological properties depend on particles polarity induced magnetic field applied on MR fluid. When there is a presence of a magnetic field, every micro iron particle is changed into a dipole and makes a chain structure with its consecutive particles which can resist failure for some ratio of heat and thus form a semi-solid structure. The interactions between the induced dipoles provoke the micro iron particles to be aligned along exposed field and make a form of column structure. The chain structure will then prevent the fluid motion and consequently rise the viscosity of the suspension[15]. Among other factors, the rheology of MR suspensions depend on, particle shape distribution, quantity of particle, properties of the constituent oil, additional additives, applied magnetic field, temperature [16]. In normal application, the off-state behavior of MR fluids relies on carrier fluid properties, additives, particle volume fraction, etc, as it shown in table 1, while the on-state performance depends on the solidity stage properties and the volume fraction of the solid stage. Rheology of MR fluids is explained in terms of pre-yield as well as post-yield conditions[17].

$\tau=\left\{\begin{array}{lr}G * \gamma_{e} & \dot{y}_{e}=0, \tau<\tau_{o} \\ \mu \dot{y}+\tau_{o} & \tau \geq \tau_{o}\end{array}\right.$

Where, $\gamma_{e}$ indicates wrench and $\mathrm{G}$ the post-yield behavior of MR fluids provided by experimental test.

Table 1. Table of rheological property of MR fluid

\begin{tabular}{cc}
\hline Property & Typical value \\
\hline Initial viscosity & $0.2-0.3[\mathrm{~Pa} . \mathrm{s}]$ at $25^{\circ} \mathrm{C}$ \\
Density & $3-4\left[\mathrm{~g} / \mathrm{cm}^{3}\right]$ \\
Magnetic field strength & $150-250[\mathrm{kA} / \mathrm{m}]$ \\
Yield point $\tau$ o & $50-100[\mathrm{kPa}]$ \\
Reaction time & 10 milliseconds \\
Typical supply voltage & $2-25 \mathrm{~V}, 1-2 \mathrm{~A}$ \\
and current & -50 to $150\left[{ }^{\circ} \mathrm{C}\right]$ \\
Work temperature &
\end{tabular}

Characteristically, the diameter size of iron particles varies between ranges of 3 to 5 microns meters. If the MR fluid is made with big size particles; it becomes difficult to stabilize the suspended particles. The cheaper iron particles widely available on different market are carbonyl iron; they are usually inhibited to sizes bigger than 1 or 2 microns. Smaller size particles provide an easy suspension. However, the manufacture process of such particles remains complex. Important smaller sized iron particles available on the market are usually oxides only, like pigments popular used in magnetic recording media[18]. In initial application where there is no presence of magnetic field MR fluids are estimated as Newtonian fluid[19]. Due to low voltage power supplies for MR fluids and relative temperature stability between $40^{\circ} \mathrm{C}$ and $+150{ }^{\circ} \mathrm{C}$, MR fluid present a great advantages more attractive smart materials than Electro Rheological fluids. MR fluids do not show yield stress, but show a variation of viscosity.

\subsection{Application of MR fluid}

Important use of MR fluids are in devices which require rapid continuity and reversible variation in rheological characteristics. Magneto-rheological devices showed a significant advantage during the last decade, since MR fluid links mechanical parts indirectly with electronic embedded controller where the control strategies are incorporated on it, thus determine continuous mechanical characteristics of device. The use of magneto-rheological fluids is advanced of dampers, clutches, brake, control valves, vehicle power steering devices, artificial joints, alternators, sound propagation, chemicals sensing applications and other application. Especially shock absorber are the commonly device which utilizes MR fluid[20].

Valves: When the magneto-rheological fluid flows into the valve and magnetic field is subjected to the fluid provoke the increment fluid viscosity. This variation of viscosity generates resistance against flow of fluid in the valve. Thereby the pressure is raised and flow reduces the speed considerably or stops completely. Rosenfeld et al investigated structure and performance of magneto-rheological and electro-rheological valves and the result showed that performance of the MR fluid significantly depends on driving force and active volume of the fluid[22].

Polishing: Optical polishing, it is a good application of MR fluid. In working operation when a magnetic field is available, magnetic particles make chain-like structure and provoke abrasive particles to increase the polishing work. The ability of abrasive to keep power and stiffness of a MR fluid is greatly dependent on the size of induced particle. Type of abrasive and concentration are the other parameters which increase the quality of piece surface.

Brakes: Magneto-rheological brake is an element which is mainly used in torque transmission. The torque of rotation MR fluids brake is changed quickly according to the magnetic field applied on it. Based on its simplicity design, ease control, the use of MR fluid remain a best choice for braking systems[23].

Dampers: Damper is an apparatus which is used to attenuate shock vibration, in addition, to mitigate vibration motion, and to minimize the kinetic force. A damper is called magneto-rheological damper when it contains magneto-rheological fluids and monitored by with variation of magnetic field. They have been designed and developed to provide damping force necessary to the suspension to mitigate any kind of unwanted vibration which occurs in many systems such as seismic, wind induced frequency, 
prosthesis control, washing machine, vehicle suspension. Whenever a problem happens in the system, the magneto-rheological damper has the ability to react as a passive damping and lithium stearate. These three components of an MR fluid ensure its magneto rheological characteristics. Modifying any one component will lead the variation of rheological and magneto rheological properties of the MR fluid. An important combination of all the three elements is important to reach the required properties of MR fluid.

\subsection{Composition of MR fluid}

The magneto rheological fluid is composed by three elements, base fluid, metal particle and additives.

Base fluid is a passive or non-magnetic carrier fluid in which the micro-sized iron particles are suspended. The base fluid must possess natural lubrication and damping specifications. In design and good application of MR fluid; base fluid should have a low viscosity and not change with temperature. It is very important that variations of viscosity caused by magnetic field become high compared to variation caused by natural physical parameters. According to the presence of suspended particles base fluid becomes dense. Famous utilized base fluids are hydrocarbon oils, mineral oils and Silicon oils[24].

Metal particle for the best used of this technology a type of metal particles which can provides a fast and easy response of magnetization is needed. Metal particles used in the MR- technology are very small. Size of the particle is around range of 1micrometer $(\mu \mathrm{m})$ to $7 \mu \mathrm{m}$. The most used and available on market metal particles are carbonyl iron, powder iron and iron cobalt alloys. The properties of these materials provide high magnetic saturation according to that they are is capable for, a good magnetizing chain structure. The concentration of magnetic particles in base fluid may reach to $50 \%$.

Additives to have a better control of MR fluid properties some amounts of additives are required. It has the role of stabilization and control the viscosity. Additives are composed by stabilizers and surfactant; surfactants ensure the decrement rate of settling of the metal particles. While the functions of additives are to control and monitoring the viscosity of the fluid, keep friction between the iron metal particles and to minimize the rate of reduction of the fluid due to long period use of the fluid consequently additives avoid wear of fluid and increase it service life[25]. Most utilized additives are ferrous oleate

\section{Magneto rheological dampers}

Based on specific application, configuration, desired load MR damper have been designed and developed in different sizes. The configuration of a damper can be classified into three main categories based on operation mode, hydraulic housing (cylinder), piston (or MR control valve) structure. Damper is composed by cylinder housing filled with the MR fluid and a control valve (piston) which generates damping force while it is in motion. Generally the control valve is embedded with solenoid which is the magnetic circuit. According to the operating principle modes MR dampers are categorized into four fundamental types: flow-mode dampers, shear-mode dampers, squeeze-mode dampers and pinch-mode dampers. Dampers which are using more than one MR fluid operation mode are called hybrid or mixed mode devices.
Every type has brought particular interest, and it might use various objectives.

\subsection{Mono tube damper}

Mono-tube dampers represent the commonly used magneto rheological devices. Due to its simplicity design and less internal parts mono tube damper remain the best choice for different application. It is widely used in passenger vehicle suspensions. Based on the standpoint of design and implementation of MR dampers, vehicle suspension using MR dampers, mono-tube presents a significant benefit of no having electromechanical valve and minimum number of moving elements. The working principle, the design setting of a characteristically gas-charged MR damper is the same as that of a natural valve-based damper. The floating piston serves on separation of MR fluid from high pressure gas chamber. The role of main piston is to separate the quantity level of magneto rheological fluid into compression chamber and rebound the fluid volume between piston road and main assembly. As it shown in Figure 6 the piston assembly embeds an annular gap (annulus) to allow the fluid to move between the chambers while in motion. The relative movement of the wheel (unsprung mass) and the body (sprung mass) conduct the fluid flow between the chambers through the annular gap in the piston. The big pressure gas in the chamber under the floating piston is needed for cavitation-free operation, but, it has an opponent effect on cylinder inner surface complete necessaries, sealing and friction[3].

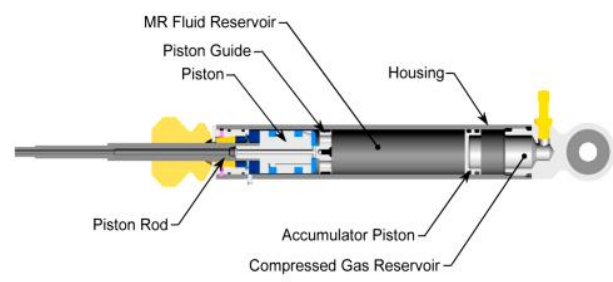

Figure 6. Mono tube MR damper

\subsection{Twin-tube Damper}

As it called the twin-tube MR damper is one of MR damper that possess two fluid reservoirs, one located inner the other one as it is indicated on the Figure7. In this type of setting, the damper possesses two housing an inner and outer housing[26]. The inner housing ensures the guidance of the piston rod assembly, in exactly the similar method as in a mono-tube damper. The volume stayed by the inner housing is assigned to as the inner reservoir. Equally, the volume that is determined by the space between the inner housing and the outer housing is assigned to as the outer reservoir. Hence no air pocket exit because inner reservoir is filled by magneto-rheological fluid[3].

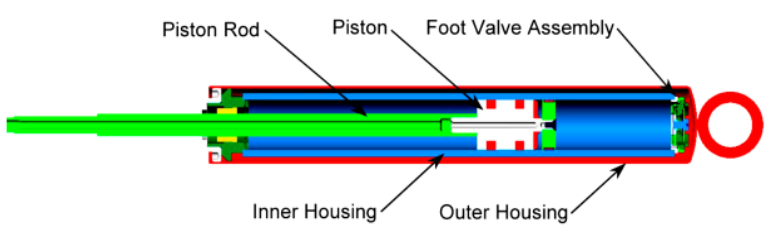

Figure 7 Twin tube MR damper 
To arrange variations in volume caused by piston motion, the use of outer reservoir partially filled is necessary. For, the outer housing in a twin-tube damper shows the similar objective as the pneumatic accumulator system in mono-tube dampers. Practically, the valve assembly named foot valve is mounted to the bottom of the inner housing to control the flow of fluid between the two tubes. Whenever the piston rod, move into the damper MR fluid move from the inner tube to the outer tube through the compression of foot valve assembly. The quantity of fluid that flows from the inner reservoir into the outer reservoir is the same as the volume moved by the piston rod as it enters the inner housing. When the piston rod is returned from the damper, MR fluid flows from the outer reservoir into the inner housing tube through the return valve, which is also part of the foot valve assembly part.

system with known operational parameters depending on specifications of the magneto rheological fluid when a magnetic field is not exposed on it[21].

\subsection{Double ended tube MR damper}

The last type of MR damper is named a double-ended damper because a piston rod of equal diameter exceeds from both ends of the damper housing. Figure8 indicates a cross section view of characteristically double ended MR damper. Because of no variations in volume exists; when the piston rod moves relatively to the damper body, in the other word the double-ended damper does not need an accumulator system. This kind of MR damper is applied in gun coil system, bicycles, seismic vibration avoidance, and wind drift[21].

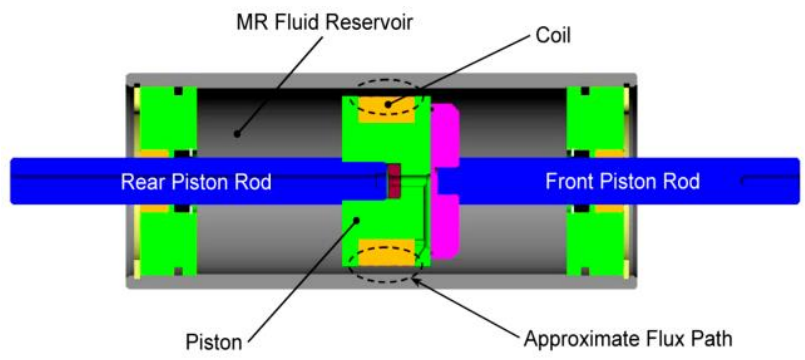

Figure 8 Double-end tube MR damper

\section{MR damper fundamental models}

The fundamental model of MR fluid is based on Bingham solid, which possess the variable yield strength (elasticity)[16]. In Bingham model, fluid flow is provided by Bingham's mathematical equations,

$\tau=\tau_{y}(H)+\eta \dot{\gamma}$

$$
\tau<\tau_{y}
$$

$\tau$ represents the fluid stress, $\tau_{y}$ denoted the field dependent yield stress, $H$ defines the magnetic field, $\dot{y}$ is defined as the fluid shear rate, and $\eta$ denotes the plastic viscosity; it means, the viscosity if $H=0$. Under the fluid's yield stress (pre-yield state), the fluid displays visco elastic characteristics[27].

\subsection{Flow mode}

The working principle of this mode is based on fluid which moves through the two fixed surfaces and magnetic field is exposed perpendicularly to the direction of flow as shown in Figure 9. By adjusting the intensity of magnetic field, the viscosity of fluid is controlled. This mode of MR fluid technology is utilized in different categories of dampers and vibration absorbers and has wide application in automotive industry. In classic dampers, fluid moves from the inner reservoir (cylinder) to outer reservoir (cylinder) through the foot valves. The air and fluid is located in outer. When piston moves down, the fluid volume level in outer cylinder raises and free air behaves as a compressed medium and generates damping force. When the piston spread out, direction of flow gets reverse MR dampers are slightly opponent from the tradition dampers. This type technology does not have the valve like classical dampers. The most application of this mode is the suspension of highway vehicles. In this kind of damper there is an annular orifice passage, through which MR fluid can be transferred from one chamber to another. In normal operation mode ( in absence of magnetic field)condition, fluid can flow through the orifice but when suspension is needed, the solenoid is energized and current starts to pass through the coils windings, and generates the intensity of magnetic field necessary to provide a damping force[24].

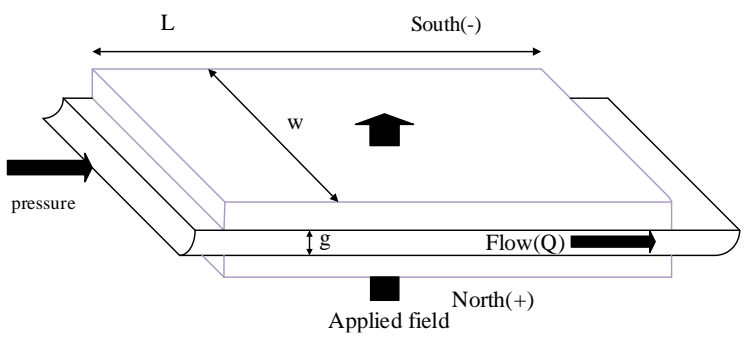

Figure 9 Flow mode

As it is shown in Figure 9 above, pressure variation in this mode is the calculated as sum of pressure generated due to fluid viscosity $\Delta P$ and pressure developed due to the magnetic field $\Delta P_{\eta}$.

$\Delta P=\Delta P_{\eta}+\Delta P_{\tau}=12 \eta Q L /\left(g^{3} w\right)+c \tau_{y} L / g$

Where $\Delta P_{\eta}$ denoted viscous component of pressure drop, $\Delta P_{\tau}$ field dependant of induced yield stress part of pressure drop, $\eta$ plastic viscosity $(H=0), \mathrm{g}$, fluid gap, W defines Width of fluid flow orifice, $Q$ pressure fluid, $\tau_{y}$ defines Field dependant yield stress found in Magneto rheological fluid, $c$, constant, L length of fluid flow orifice.

\subsection{Shear mode}

The operation of this mode is based on the fluid which moves between surfaces having same motion and a magnetic field is subjected perpendicular to the direction of flow as shown in Figure 10 [28]. Shear mode of MR fluid technology is utilized in different type of brakes and clutches of automotive. In this mode, the total shear force generated is a summation of the force generated based on viscosity of the fluid $\left(F_{v}\right)$ and the force generated based on the magnetic field $\left(F_{m}\right)$. 
$\sum F=F_{v}+F_{m}$

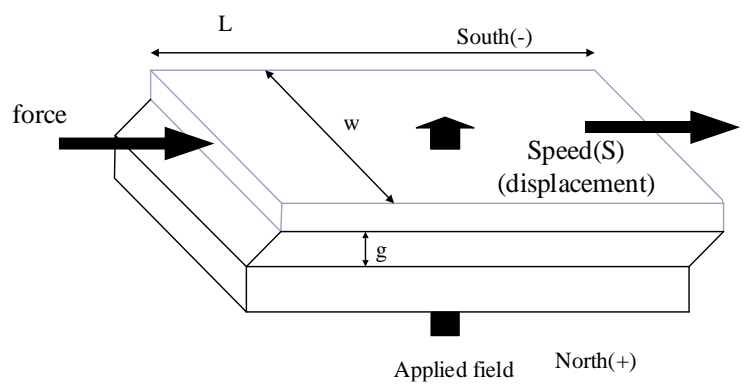

Figure 10 shear mode

The design of squeeze mode Figure 11 is simple and possesses the minimum moving elements hence, less maintenance is desired, and service life is increased. The main disadvantage of MR fluid technology is that the MR fluid becomes dense after elongated use and needs to be changed, also the weight of MR fluid is high caused based the high density of iron metal particles. Presently automotive industries are applying this system. The application of this technology is wide such as, medical engineering, aerospace and polishing. There is a large range of research in MR fluid technology. MRF systems have to be designed more delicate, probably by introducing the uses of sensors and feedback technology i.e. closed loop systems. The life range of MR fluid devices in terms of the total energy dissipated from the equipment need to be increased. An improved MRF technology would make it the smart technology of future[28].

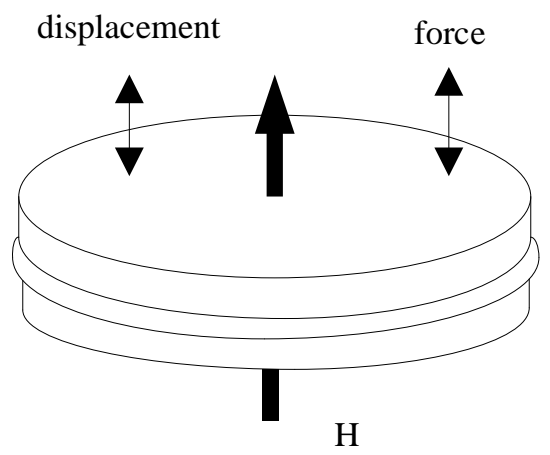

Figure 11 Squeeze mode

\section{Mechanical model of MR damper}

MR dampers are called semi-active elements of suspension systems whose damping specifications may be changed in real time based on the ability to change the viscosity of MR fluid as well its resistance to circulate by using of magnetic field. The benefit of MR fluid is that can permit the changes in the damping force by varying the current subjected to the damper. Thereby the hysteretic model characteristics of MR damper are based on current dependent and a function of excitation amplitude. It is important to analyze and model the MR damper with appropriate methods in order to predict it behavior in various magnetic fields or excitations. This approach becomes important aspect because before the production of a MR damper it is significant to design and choose the right parameters that will reach the behavior of the damper[29].

\subsection{Bingham model}

This Bingham model investigates and study plastic characteristic and the behavior of magneto-rheological and electro rheological fluids. Bingham model ensure that a body remain as solid till a little yield stress is surpassed then after it shows a linear relationship between the stress and the rate of shear deformation[30]. Some researchers like Stanway et al. investigated a mechanical model based on the Bingham plastic model to exhibits the ER damping system. As it indicated in Figure 12 the model is based on a combination between coulomb friction and viscous dashpot. and is always utilized to analyze the characteristic or behavior of MR dampers[31].

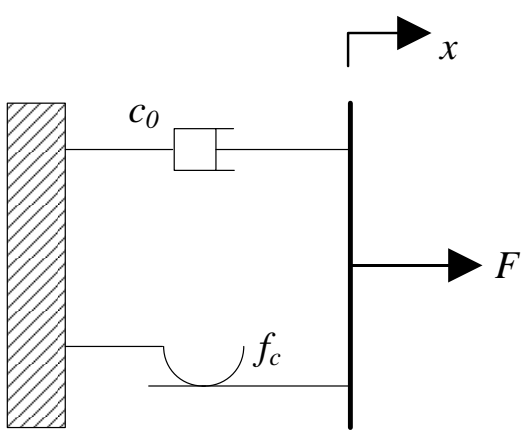

Figure 12 Bingham model

The force provided by mechanical element setting is coming from equilibrium concepts; it is expressed in the equation below:

$F(t)=c_{0} \dot{x}+f_{c} \operatorname{sgn}(\dot{x})+f_{0}$

Where, $c_{0}$ denoted as constant of damping coefficient, $\dot{x}$ defines the velocity of the external excitation fc denoted the frictional force and $f_{0}$ is the force offset related with the availability of an accumulator considering that this element has linear behavior and low stiffness. To investigate the flow of fluid, this mathematic expression model can be derived.

\subsection{Bouc-Wen model}

The hysteresis Bouc-Wen equation is among different models and the most used to study and simulate various method of expanding the damping system by changing the hysteresis. It has been invented by Bouc in 1971[32] and later developed by Wen in 1976[33] who has showed different methodologies of this model to show a wide kind of hysteretic patterns. According to this interesting behavior, the model was utilized to analyze different nonlinear hysteretic systems like MR damper and hysteretic isolators. However, MR dampers show a good typical behavior, the final hysteretic setting relies on certain particular specification features related to accumulator, damper geometry and so, on. So, the model should be suitable to have the real MR damper characteristic and several changes of the Bouc-wen model were designed and developed to simulate properly MR damper. Based on BoucWen model different procedure to study the simulation the MR damper has been proposed; these models are: Classic or simple Bouc-Wen model; 
(1) Advanced Bouc-Wen model or Spencer model;

(2) Shear model Bouc-Wen model

(3) Bouc-Wen model for wide scale MR dampers;

(4) Inverse Bouc-Wen model which is current dependant;

(5) Current-frequency-amplitude-dependent;

(6) Asymmetrical Bouc-Wen model.

As, it is indicated in the Figure13, the simple Bouc-Wen model is composed by three elements: a spring, a Bouc-Wen block and dashpot, mounted a parallel setting. In my project the simple model of Bouc-Wen will be used to simulate the MR damper behaviors. In this figure nonlinearity of the system is situated in the Bouc-Wen block, which is able to track the behavior of MR damper:

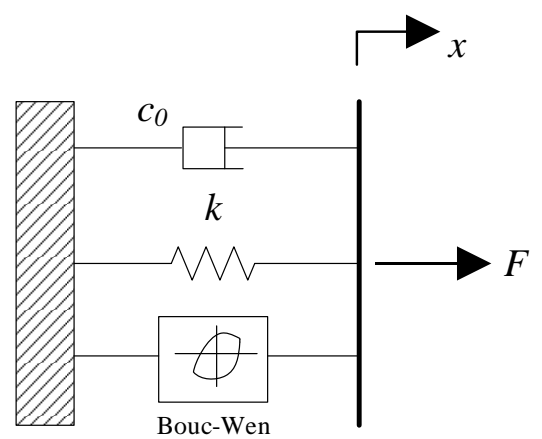

Figure 13 Bouc-wen model

The model investigates different characteristics of MR damper by comparing the performance of this model with other parametric models. Based to mechanical setting indicated in Figure13, the equation below express the damping force.

$F(t)=c_{0} \dot{x}+k\left(x-x_{0}\right)+\alpha z$

Where $c_{0}$ denoted the constant of viscous coefficient, $k$ denoted the constant of stiffness coefficient and $z$ is an evolutionary related variable with Bouc-Wen block and it ruled by:

$\dot{z}=-\gamma|\dot{x}| z|z|^{n-1}-\beta \dot{x}|z|^{n}+A \dot{x}$

$x_{0}$ is denoted the displacement at initial state, which permit the presence accumulator in the mechanism system. Constant $c_{0}$, $k, \alpha, \beta, \gamma, A$ and $n$ are generally and the Bouc-Wen shape parameters or behaviors. They are the functions of the current subjected to the MR damper, frequency of vibration and amplitude. Nonlinear shape hysteretic curve can be controlled by varying the amounts of the Bouc-Wen block parameters permitting to monitor the smoothness in the state of transition between pre yield and post yield. Based to the ability to take the hysteretic element of the MR damper characteristics, the classic Bouc-Wen model is considered a best rational model of mathematical and numerical simulation compare to Bingham model. It is better in analysis of hysteric behavior. The use of Bouc-Wen hysteric model, lead on a good simulation of MR damper, test and implementation of its parties[35].

\section{Semi-active vehicle suspension control strategies}

Semi-active suspension is considered as the union of active and passive suspension. Since its invention, the damping force was adjusted by the variation of orifice area which leads on the change of flow resistance. MR damper is a kind of semi-active damper where the motion of MR fluid is adjusted by changing the value of current applied and thereby varies the level of damping force. As other control strategies of semi-active suspension dampers the MR damper is capable to provide a damping system even if the current become zero. The damper is able of generating huge control forces by changing behaviors like stiffness and damping coefficient through the variation of magnetic field and thus controls the response of large scaled structures like big buildings [36]. Control strategies of this system are to give a good stability handling and a smooth riding. Many studies and researches have been conducted to optimize the trade-off between road handling and ride comfort. These control strategies are categorized into techniques based on, nonlinear, linear and intelligent control methodologies.

\subsection{Skyhook control}

Skyhook control strategy is among the semi-active control strategies and the most commonly utilized that changes the damping force to imitate the damping effect of a passive damper in its optimal setting, mounted between the fixed reference and sprung mass (vehicle body) as showed in Figure 14 [58].

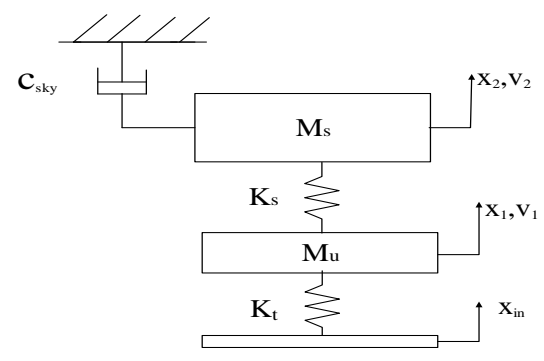

Figure14 skyhook control strategy

It is characteristically categorized as continuous control skyhook and on-off skyhook system. On-off Skyhook controller is always easier designed and well appropriated for the industrial era [37]. To implement the control strategies of On-off law equation below should be governed.

$C_{\text {sky }}= \begin{cases}C_{\max } & \text { if } V_{r} V_{2} \geq 0 \\ C_{\min } & \text { otherwise }\end{cases}$

Where, $V_{2}$ is defined the absolute velocity of the body, and $V_{r}$ denoted as the relative velocity of the sprung mass (body) and unsprung mass (wheel) across the suspension. It is explained that if relative velocity of sprung mass (body) and the wheel have the similar direction, Hence, the maximum damping force must be subjected to mitigate the body acceleration. Meanwhile, if the two velocities (sprung velocity and unsprung velocity) are in the odd directions, the damping force must be set at low level to mitigate body acceleration. To achieve the skyhook controller strategy, absolute velocity of the body should be measured. Skyhook strategy 
has been proven the optimization of ride behavior by minimizing vertical body acceleration. Skyhook control is good approach to minimize the vertical vehicle dynamic. But, according to its emphasis on optimizing a smooth ride, the road handling performance is very small. To prevent this issue ground-hook control strategy has been designed and proposed to optimize road handling abilities for controllable suspensions. The theory is based on the virtual damper which mounted on a fixed ground instead of sky which will give consideration to road holding ability to suspension mechanism[38].By comparing the skyhook semi active controller with passive suspension; models are simulated using a square bump as road profile, results show that the skyhook presents a great contribution to comfort of vehicle occupants as it is shown to the figure. 15 Where minimum body acceleration is indicating ride comfort

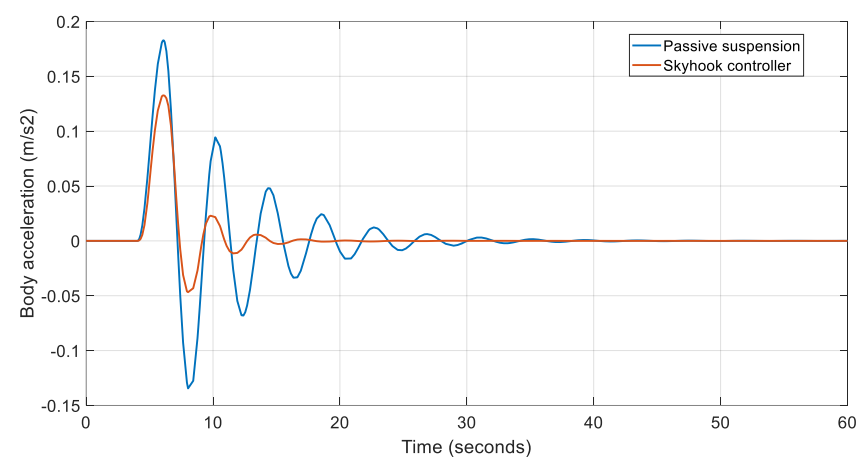

Fig: 15. Body acceleration comparison skyhook vs Passive

\subsection{Ground hook control}

As it is indicated in the Figure 16, the ground hook strategy is opposite from the skyhook model. In this model, the damper is mounted to the wheel rather than the body. Under the ground hook setting, the focus changes from the sprung-mass to the unsprungmass [60].

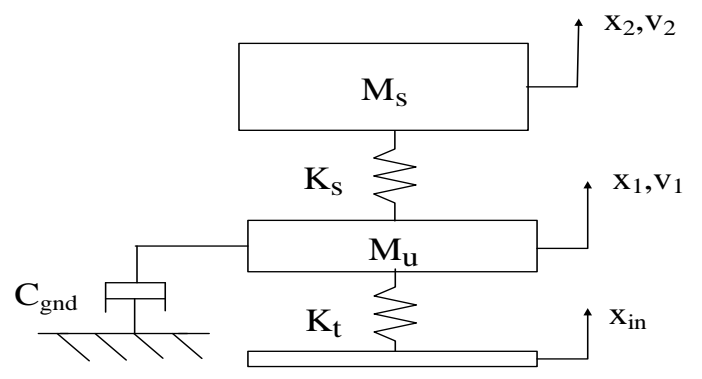

Figure 16.Ground hook strategies

As it indicated in this Figure16, in this setting the focus shifts from the sprung mass to unsprung mass. While skyhook work well in controlling the vertical body mass, ground hook is good in controlling the unsprung mass frequencies. The idea of ground hook is the same as the skyhook strategy, the difference is that it is ground hook control the wheel vibration [39].

$$
\left\{\begin{array}{cc}
-V_{1} V_{2} \geq 0 & F_{s a}=C_{g n d} V_{1} \\
-V_{1} V_{2}<0 & F_{s a}=0
\end{array}\right.
$$

\subsection{Sliding control mode}

Sliding control mode is a strong control strategy with various significant functionalities like insensitivity to linked behavior changes and disturbance isolation. Depending on its applications, sliding mode controller design showed a great potential in keeping the stability and optimization of in modeling and simulation the uncertainties. Sliding controller is constituted by of a nominal part, the same closed loop theorem, and additional conditions objected to face with uncertainty model. Reference model of Sliding Model Control is consist on forming a 'sliding surface' to track errors, the tracking should be equivalent all time[40].

Sliding-mode design has various functionalities that make it an advantageous option to remove nonlinearity tracking process, however; it presents a great importance in designing and modeling of uncertainties systems. It can be explained in many ways [41]. The first way is to consider that the system to process has two step procedures. Primo is the sliding surface which the desired state of the system. Secondly, a control system to conduct toward a sliding surface in the other word a system to track the sliding surface system, then after switching on that surface. In theory sliding mode will effectively approach the control purpose, the cause of phase surface[42]. The control action design can be pretended according on various strategies, a straightforward one which is based on determining a Lyapunov-like condition that make the sliding surface to be an attractive region for the state vector trajectories[43].

Reference: the idea of reference is based on development of control algorithm necessary to force the plant to virtual dynamic model. So, to consider a reference model is very useful. In model reference control for instance a set point regulation problem is changed into a tracking problem by immerging a reference model to filter the given configuration point value and to provide output which is variable with time as virtual response of tracking control mechanism[42].

Based to the sliding mode control (SMC) regulation, as it is indicated on the Figure $17 \mathrm{a}$ and $\mathrm{b}$, an asymptotically fixed sliding mode state can be derived from dynamic errors between the MR quarter suspension of vehicle dynamic system and the transformed skyhook reference model approach[44].

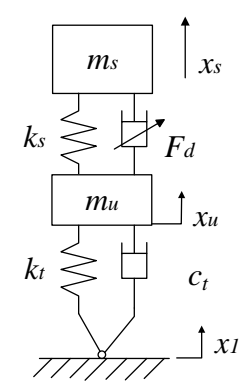

(a) Quarter car model

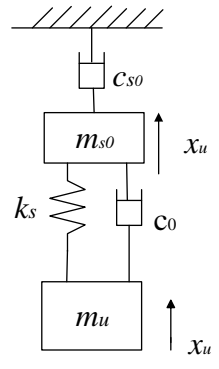

(b) Modified skyhook reference model
Figure 17. Sliding control mode

According to skyhook reference model wheel (unsprung mass), the displacement $x_{u}$ of MR quarter-vehicle suspension can be directly utilized as road variation input of the skyhook reference model system, because the real tire stiffness is typically 5 times of the suspension spring stiffness. Based on hypothesis 
above the dynamic equation of skyhook reference is expressed as[45]:

$m_{s 0} \ddot{x}_{s 0}+c_{0}\left(\dot{x}_{s 0}-\dot{x}_{u}\right)+c_{s 0} \dot{x}_{s 0}+k_{s}\left(x_{s 0}-x_{u}\right)=0$

Where $m_{s 0}$ denoted as reference model sprung mass, which is equivalent to unloaded sprung mass of the semi-active quarter-vehicle suspension, $c_{s 0}$ denotes skyhook damping constant, and $x_{s 0}$ are defined as body mass displacement of reference model, $k_{s}$ and $c_{0}$ defined as spring stiffness of the passive quarter-vehicle suspension and damping constant coefficient, respectively. The sliding mode $s$ of MR quarter-vehicle system is expressed as this equation below:

$s=\dot{e}_{s}(t)+\lambda e_{s}(t)$

where $\lambda>0$ defines the convergence rate of sliding mode surface, $e_{s}(t)=x_{s}(t)-x_{s 0}(t)$ defines the errors between body mass displacement of MR suspension quarter car dynamic and dynamic system of skyhook reference and $e(t)$ become 0 if $t$ tends to infinite. Moreover, Lyapunov function is expressed as $V=s^{2} / 2$. To make sure that the system state asymptotically adjusts to the sliding surface mode, it is denoted that

$$
\dot{V}=\dot{s} s \leq-\phi|s|
$$

Where $\phi$, is denoted as positive constant. If the effect of gravity is neglected, the equation (13), is gotten

$\left\{\begin{array}{l}m_{s} \ddot{x}_{s}+k_{s}\left(x_{s}-x_{u}\right)+F_{d}-m_{s} g=0 \\ m_{u} \ddot{x}_{u}+F_{t}-k_{s}\left(x_{s}-x_{u}\right)-F_{d}-m_{u} g=0\end{array}\right.$

We get

$\ddot{x}_{s}=\left[-k_{s}\left(x_{s}-x_{u}\right)-F_{d}\right] / m_{s}$

By combining Eq. (13) and Eq. (14), equation (15) is obtained

$\dot{s}=\left[-k_{s}\left(x_{s}-x_{u}\right)-F_{d}\right] / m_{s}-\ddot{x}_{s 0}+\lambda \dot{e}_{s}(t)$

Base on the stability functionality of sliding mode control strategy, $\dot{\mathrm{s}} s=0$, the virtual control damping force $F \mathrm{~d} 0$ of $\mathrm{MR}$ quarter car suspension can be derived from Eq. (15)

$$
F_{d 0}=-k_{s}\left(x_{s}-x_{u}\right)-m_{s 0} \ddot{x}_{s 0}+m_{s 0} \lambda \dot{e}_{s}
$$

To guarantee that yielded damping force $F_{d}$ of the MR damper can quickly track its virtual control damping forces $F_{d 0}$, by using switch control law, the damping control force is denoted

$F_{c}=F_{d 0}-K \cdot \operatorname{sgn}(s)$

Where $K$ defines the constant gain of SMC which will be derived later and $\operatorname{sgn}(s)$ is limit function which vary between 1 and 1 , If $s \leq 0$ and $s>0$, respectively. Based on $V=\dot{\mathrm{s}} s, F_{c}$ to replace $F_{d}$ in Eq. (15) integrate it into $V=\dot{\mathrm{s}} S$ and therefore, obtain $\dot{V}$ is gotten

$\dot{V}=\left(-k_{s} x_{s} / m_{s}+k_{s} x_{u} / m_{s}-F_{c} / m_{s}-\dot{x}_{s 0}+\lambda \dot{e}_{s}\right) s$

Combining Eq. (16) and (18) $\dot{V}$, is obtained

$\dot{V}=\left[\left(1 / m_{s}-1 / m_{s 0}\right)\left(-k_{s} x_{s}+k_{s} x_{u}-F_{d 0}\right)+K \cdot \operatorname{sgn}(s) / m_{s}\right]$

Based on the Lyapunov stability of Sliding control mode, Eq. (19) is integrated into Eq. (14) and obtain $\left[\begin{array}{c}\left(\frac{1}{m_{s}}-\frac{1}{m_{s 0}}\right)\left(-k_{s} x_{s}+k_{s} x_{s}-F_{d 0}\right) \\ +K \cdot \operatorname{sgn}(s) / m_{s}\end{array}\right] s \leq-\emptyset|s|$

As considering $s=s^{*} \operatorname{sgn}(s)$, Hence Eq. (20) is governed as

$\left[\begin{array}{c}\left(\frac{1}{m_{s}}-\frac{1}{m_{s 0}}\right)\left(-k_{s} x_{s}+k_{s} x_{u}-F_{d 0}\right) \\ +K \cdot \operatorname{sgn}(s) / m_{s}\end{array}\right] s \leq-\emptyset \cdot s \cdot \operatorname{sgn}(s)$

for $s \geq 0$, Eq. (21) can be simplified as

$K \leq-m_{s} \varnothing+\left(1-m_{s} / m_{s 0}\right)\left(k_{s} x_{s}-k_{s} x_{u}+F_{d 0}\right)$

for $s<0$, Eq. (21) can be simplified as

$K \leq-m_{s} \emptyset+\left(1-m_{s} / m_{s 0}\right)\left(-k_{s} x_{s}+k_{s} x_{u}-F_{d 0}\right)$

If the real load of vehicle is considered as usually variable $\eta$ is expressed as the change rate of vehicle body mass (sprung mass) $m_{s}$ to its unloaded sprung mass $m_{s 0}$, in the other word $s=m_{s} / m_{s 0} \geq 1$. By using Eq. (22) and (23), $K$ of sliding control mode is obtained

$K=-m_{s 0} \eta \emptyset-(\eta-1)\left(\left|F_{d 0}\right|+k_{s}\left|x_{s}\right|+k_{s}\left|x_{u}\right|\right)$

Therefore, the saturation function $\operatorname{sat}(s)$ is used to replace the sign function $\operatorname{sgn}(s)$ in Eq. (20) for limiting chattering frequency which may happen in the system, and takes $s / \varepsilon$ and $\operatorname{sgn}(s)$ when $s \leq \varepsilon$ and $s>\varepsilon$, accordingly. Eq. (20) can be shown as

$F_{c}=F_{d 0}-\operatorname{Ksat}(s)$

Where $\varepsilon$ denotes positive constant. Fig18, indicates the ride comfort between passive suspension and semi active suspension in sliding mode control strategy. The improvement is more than $35 \%$ compare to passive suspension.

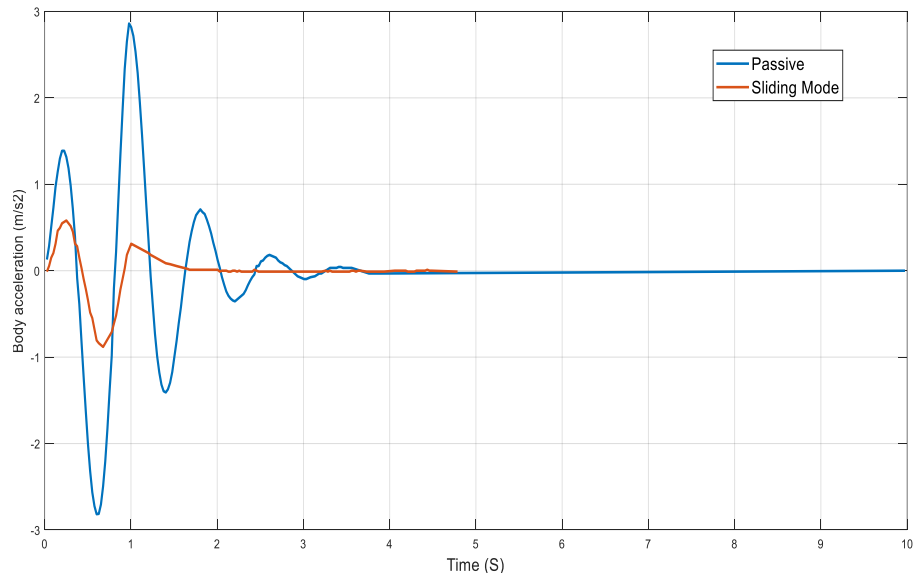

Figure18. Body acceleration comparison of sliding mode and passive

\subsection{Fuzzy logic control strategy}

Fuzzy logic control strategy is a type of many values of logic. In many applications of control of nonlinear systems, this approach developed by mathematicians has been appreciated and successful used. Since the last decades the implementation of fuzzy logic has been growth due to the various researches which showed its potential on controlling the nonlinearities systems, 
Fuzzy logic has been inverted by Zadeh [46] in 1965 , ten year after its invention, Mamdani [47] developed and showed the utility of Fuzzy Logic Control (FLC),in modeling and control of small steam engine's suspension to replace the tradition suspension of damper and spring of vehicle by active suspension. The result of Fuzzy logic model demonstrated a big improvement of riding comfort and road handling in various conditions [1]. Presently fuzzy logical is applied in many areas such as dynamic process, washing machine and in automation industries. The theory of fuzzy control is based on operations rules that are human can read and represent human's knowledge about how to control and monitoring the process. The rules are defined in terms of fuzzy involvement. It is composed by three main elements namely input, process output and a reference signal. For the suspension vertical dynamics application, input is the suspension damping control and process output is a rate of velocities between sprung and unsprung masses [49].

Generally, input is supplied to Fuzzifier which changes crisp data input into fuzzified data which is then analyzed processed in Fuzzy inference function block using fuzzy rules. This fuzzy output is then converted to crisp output using Defuzzfier. Fig 19 indicates schematic of Fuzzy control methodology which is the flowchart of control strategy. Fuzzy logic approach control has many features that make it attractive for semi-active suspension. There are a many nonlinear devices like tires shock absorbers springs and many rubber components. All these non-linearities can be made linear having significant impact on the precision of model.

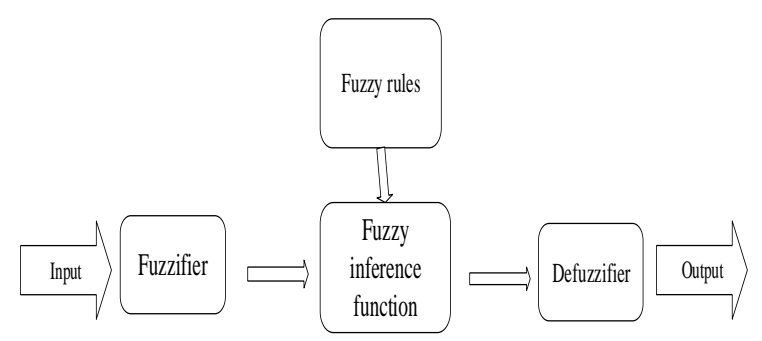

Figure 19 fuzzy control methodology

Fuzzy logic algorithm controls many loops variation in various non-linear systems. it has two inputs, namely suspension deflection and suspension velocity. the controller calculates those inputs and generate the desired damping force based on inference rules subjected on it. These control systems embed the human as thinking through the application of fuzzy configurations and linguistic variables related by a configuration of IF-THEN fuzzy rules. The results show figure 20 and 21 that fuzzy logic can dramatically reduce the tradeoff between road handling and ride comfort. However, it can't completely mitigate this odd.

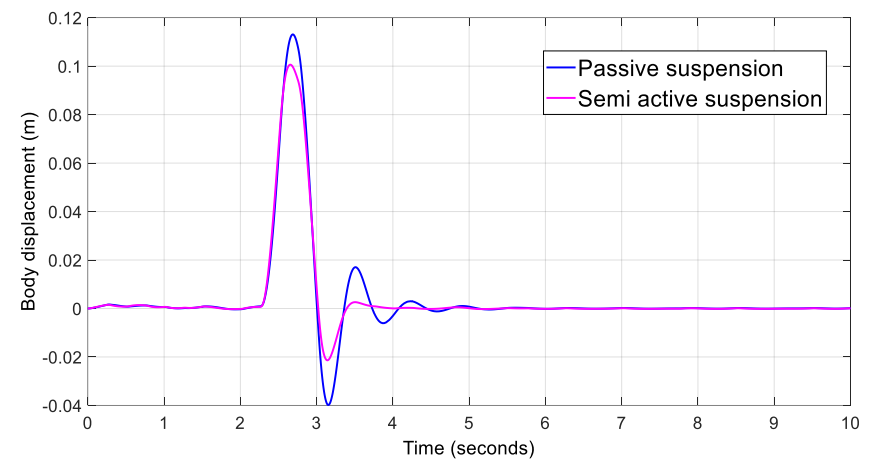

Figure 20 Body acceleration comparison of semi active and passive

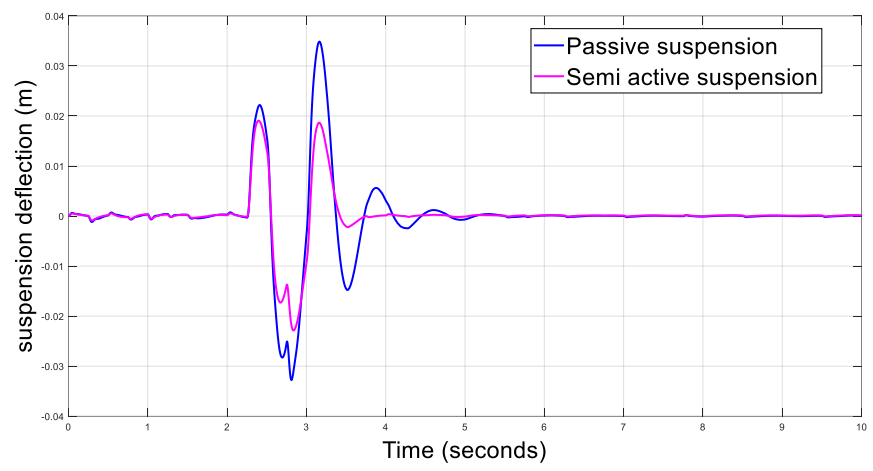

Figure21 Suspension deflection comparison of semi active and passive

\subsection{Linear Quadratic Gaussian}

Linear-Quadratic-Gaussian (LQG) control is a robust strategy which is based on an advanced state-space algorithm to design optimal dynamic regulators. It is based on noise measurement of the process and requires a state-space model of the plant[50]. This control algorithm was less used in control of semi-active vehicle suspension based on MR dampers, thus it was largely applied in control and monitoring of active suspension to mitigate the vibrations. To examine the effectiveness of semi active suspension controller, the LQG control strategy is utilized to calculate the desired damping force $f_{d}$ necessary to generate a smooth ride and comfort. LQG is composed by Kalman filter gain $K_{f}$ and of an optimal statefeedback, gain $K$ named Linear-Quadratic-Regulator (LQR). The optimal control has been designed to find a controller which may perform better. The optimal controller LQR for the vehicle suspension control is the most utilized in background of various researches of vehicle suspension control. The strength of LQR method is that in using it the factors of the optimization index can be weighted based to the designer's wish or other constraints. The LQR approach is utilized to optimize the road holding and the drive comfort for vehicle suspension. LQR control approach remain better in control of linear active suspension mechanism [50].

In modeling and control of linear active suspension, the equation below denoted the system

$\dot{x}(t)=A x(t)+B u(t)+f(t)$

$A$ state variable of feedback regulator of the system is considered as 
$u(t)=-K x(t)$

$K$ denotes the state feedback constant gain matrix (controller). Hence, a new system is obtained as

$\dot{x}(t)=(A-B K) X(t)+f(t)$

The perform procedure serves on defining input of control $U$, which reduce the performance index. $J$ is performance index show the optimization required behavior as well as the controller input mitigation. The LQR optimal controller of provided system is defined as controller design which reduces the performance index below.

$J=\int_{0}^{\infty}\left(X(t)^{T} Q X(t)+u(t)^{T} R u(t)\right) d t$

Where u defines state input, $R$ and $Q$ denoted the positive weighting matrices.

Controller gain $K$ is defined by:

$K=R^{-1} B^{T} P$

Where $P$, is denoted as the matrix which is studied as solution of the Algebraic Riccati Equation (ARE) as follow as $P A+A^{T} P-P B R^{-1} B^{T} P+Q=0$

To evaluate the Kalman filter gain $K f$, the solution of the following Algebric Riccati Equation is obtained as $S$

$A S+S A^{T}+G W G^{T}-S C^{T} V^{-1} C S+Q=0$

Then, calculating $K_{f}$ like that,

$K_{f}=S C^{T} V^{-1}$

The Kalman filter for the LQR controller becomes

$\dot{\hat{x}}(t)=A \hat{x}(t)+B u(t)+K_{f}[y(t)-C \hat{x}(t)-D u(t)]$

The equation below is considered as system

$\dot{x}=A x+B f_{a}+G w$

Where $w(t)$ is defines as a fixed Gaussian white noise process. Then, system (29) is denotes as linear system applied to a Gaussian noise. By solving stochastic equation of optimal control issue, and measuring the feedback system the equation (36) is gotten.

$Y=C x(t)+D u(t)+v(t)$

Where $v(t)$ defines a random signal which defines the measurement noise. The plant and measurement noise are remained both white noise and simultaneous. As it is shown below,

$E\left\{w w^{t}\right\}=W, E\left\{v v^{t}\right\}=V, E\left\{w v^{t}\right\}=0$

The LQR gain $K$ defined above are utilized with a Kalman filter gain $K_{f}$ to get state estimator. It was determined that the body acceleration $\ddot{x}_{b}$ and the wheel acceleration $\ddot{x}_{w}$ measurements are available in addition to the suspension working space $x$. In other words, the output vector is assumed to be $Y=\left[x_{b}-x_{w}, \ddot{x}_{b}, \ddot{x}_{w}\right]$. The measured parameters are utilized to optimize LQG controller of suspension because the cost accelerometers are relatively low, and LQ was not robust enough to perform a better control.

\section{Conclusion}

Semi active control strategies based on Magneto-rheological damper are studied and reviewed in details, through the Boucwen model for MR damper, the simulation was conducted based on quarter car model passing on trapezoidal bump, the control improvements are just subjective and controllers should act with a compromise between odds behaviors which is ride comfort and vehicle stability. Simulation results for various control strategies like skyhook, groundhook, slide mode controller, fuzzy logic and Linear quadratic Gaussian for Bouc-Wen magne-torheological damper, showed that the semi active suspension acts well on the reduction of compromise between comfort and stability, however it can't mitigate this compromise at $100 \%$ this odd. The authors suggest that combining more than two control strategies could dramatically reduce this compromise issue between comfort and vehicle stability. For the future scope the use of artificial neuronetworks in control strategy could bring lower odd between ride comfort and road handling.

\section{Conflict of Interest Statement}

The authors declare that there is no conflict of interest.

\section{CRediT Author Statement}

Musabyimana Josee: Supervision, Methodology, article editing, Kazima Sosthene:, Writing original draft and revision, Turabimana Pacifique: Writing original draft and revision

\section{References}

1. Aly, A. A., \& Salem, F. A. (2013). Vehicle suspension systems control: a review. International journal of control, automation and systems, 2(2), 46-54.

2. Rao, T. R. M. R., Mohan, R., Rao, G. V., Rao, K. S., \& Purushottam, A. (2010). Analysis of passive and semi active controlled suspension systems for ride comfort in an omnibus passing over a speed bump. International Journal of Research and Reviews in Applied Sciences,5(1).

3. Ahmadian, M. (2014). Integrating electromechanical systems in commercial vehicles for improved handling, stability, and comfort. SAE International Journal of Commercial Vehicles, 7(2014-01-2408), 535-587.

4. Fialho, I., \& Balas, G. J. (2002). Road adaptive active suspension design using linear parameter-varying gain-scheduling. IEEE transactions on control systems technology, 10(1), 43-54.

5. Mulla, A. A., \& Unaune, D. R. (2013, March). Active suspensions future trend of automotive suspensions. In International Conference on Emerging Trends in Technology\&Its Appliocations, ICETTA.

6. Gysen, B. L., Paulides, J. J., Janssen, J. L., \& Lomonova, E. A. (2009). Active electromagnetic suspension system for improved vehicle dynamics. IEEE Transactions on Vehicular Technology, 59(3), 11561163.

7. Rashid, M. M., Hussain, M. A., Rahim, A. N., \& Momoh, M. J. E. (2007). Development of a semi-active car suspension control system using magneto-rheological damper model.

8. Félix-Herrán, L. C., de Jesús Rodríguez-Ortiz, J., Soto, R., \& Ramírez-Mendoza, R. (2008, October). Modeling and control for a 
semi-active suspension with a magnetorheological damper including the actuator dynamics. In 2008 Electronics, Robotics and Automotive Mechanics Conference (CERMA'08) (pp. 338-343). IEEE.

9. Rabinow, J. (1948). The magnetic fluid clutch. Electrical Engineering, 67(12), 1167-1167.

10. Kim, J. H., Kang, B. W., Park, K. M., Choi, S. B., \& Kim, K. S. (2002). MR inserts for shock wave reduction in warship structures. Journal of 29. intelligent material systems and structures, 13(10), 661-665.

11. Seong, M. S., Choi, S. B., \& Sung, K. G. (2011). Control strategies for vehicle suspension system featuring magnetorheological (MR) damper. Vibration Analysis and Control: New Trends and Developments; InTech: London, UK, 97-114.

12. Sosthene, K., Josee, M., \& Xiong, H. U. İ. (2018). Fuzzy logic controller for semi active suspension based on magneto-rheological damper. International Journal of Automotive Engineering and Technologies, 7(1), 38-47.

13. Carlson, J. D., Catanzarite, D. M., \& St. Clair, K. A. (1996). Commercial magneto-rheological fluid devices. International Journal of Modern Physics B, 10(23n24), 2857-2865.

14. Ashtiani, M., Hashemabadi, S. H., \& Ghaffari, A. (2015). A review on the magnetorheological fluid preparation and stabilization. Journal of magnetism and Magnetic Materials, 374, 716-730.

15. Muhammad, A., Yao, X. L., \& Deng, Z. C. (2006). Review of magnetorheological (MR) fluids and its applications in vibration control. Journal of Marine Science and Application, 5(3), 17-29.

16. Jolly, M. R., Bender, J. W., \& Carlson, J. D. (1999). Properties and applications of commercial magnetorheological fluids. Journal of intelligent material systems and structures, 10(1), 5-13.

17. Gołdasz, J., \& Sapiński, B. (2015). Insight into magnetorheological shock absorbers (pp. 1-224). Switzerland: Springer International Publishing.

18. Goncalves, F. D., Koo, J. H., \& Ahmadian, M. (2006). A review of the state of the art in magnetorheological fluid technologies--Part I: MR fluid and MR fluid models. The Shock and Vibration Digest, 38(3), 203-220.

19. Kciuk, M., \& Turczyn, R. (2006). Properties and application of magnetorheological fluids. Journal of Achievements in Materials and Manufacturing Engineering, 18(1-2), 127-130.

20. Hajalilou, A., Mazlan, S. A., Lavvafi, H., \& Shameli, K. (2016). Magnetorheological fluid applications. In Field Responsive Fluids as Smart Materials (pp. 67-81). Springer, Singapore.

21. Yao, G. Z., Yap, F. F., Chen, G., Li, W., \& Yeo, S. H. (2002). MR damper and its application for semi-active control of vehicle suspension system. Mechatronics, 12(7), 963-973.

22. Olabi, A. G., \& Grunwald, A. (2007). Design and application of magneto-rheological fluid. Materials \& design, 28(10), 2658-2664.

23. Gudmundsson, K. H., Jonsdottir, F., \& Thorsteinsson, F. (2010). A geometrical optimization of a magneto-rheological rotary brake in a prosthetic knee. Smart materials and Structures, 19(3), 035023.

24. Baranwal, D., \& Deshmukh, T. S. (2012). MR-fluid technology and its application-a review. International Journal of Emerging Technology and Advanced Engineering, 2(12), 563-569.

25. De Vicente, J., Klingenberg, D. J., \& Hidalgo-Alvarez, R. (2011). Magnetorheological fluids: a review. Soft matter, 7(8), 3701-3710.

26. Dyke, S. J., Spencer Jr, B. F., Sain, M. K., \& Carlson, J. D. (1996). Modeling and control of magnetorheological dampers for seismic response reduction. Smart materials and structures, 5(5), 565.

27. Carlson, J. D., \& Jolly, M. R. (2000). MR fluid, foam and elastomer devices. mechatronics, 10(4-5), 555-569.

28. Gong, X., Ruan, X., Xuan, S., Yan, Q., \& Deng, H. (2014). Magnetorheological damper working in squeeze mode. Advances in Mechanical Engineering, 6, 410158.

. Braz-César, M. T., \& Barros, R. (2012). Properties and numerical modeling of MR dampers. Proceedings of the ICEM15-Experimental Mechanics: New trends and Perspectives, 1199-1200.

30. Stanway, R. S. J. L., Sproston, J. L., \& Stevens, N. G. (1987). Nonlinear modelling of an electro-rheological vibration damper. Journal of Electrostatics, 20(2), 167-184.

31. Guo, S., Yang, S., \& Pan, C. (2006). Dynamic modeling of magnetorheological damper behaviors. Journal of Intelligent material systems and structures, 17(1), 3-14.

32. Bouc, R. (1971). A mathematical model for hysteresis. Acta Acustica united with Acustica, 24(1), 16-25.

33. Wen, Y. K. (1976). Method for random vibration of hysteretic systems. Journal of the engineering mechanics division, 102(2), 249263.

34. Spencer Jr, B., Dyke, S. J., Sain, M. K., \& Carlson, J. (1997). Phenomenological model for magnetorheological dampers. Journal of engineering mechanics, 123(3), 230-238.

35. Sosthene, K., JOSEE, M., \& EMMANUEL, K. (2018). Vehicle ride comfort optimization based on Magneto-rheological damper. International Journal of Automotive Science And Technology, 2(4), 1-8.

36. Y. Iskandarani and H. Karimi, "Hysteresis modeling for the rotational magnetorheological damper," in Proceedings of the 4th WSEAS International Conference on Energy and Development-environmentbiomedicine, 2011, pp. 479-485.

37. Zhang, H., Winner, H., \& Li, W. (2009). Comparison between skyhook and minimax control strategies for semi-active suspension system. World Academy of Science, Engineering and Technology, 55(5), 618-621.

38. Choi, S. B., Seong, M. S., \& Kim, K. S. (2009). Vibration control of an electrorheological fluid-based suspension system with an energy regenerative mechanism. Proceedings of the Institution of Mechanical Engineers, Part D: Journal of Automobile Engineering, 223(4), 459-469.

39. Mulla, A., Jalwadi, S., \& Unaune, D. (2014). Performance analysis of skyhook, groundhook and hybrid control strategies on semiactive suspension system. International Journal of Current Engineering and Technology, 3, 265-269.

40. Assadsangabi, B., Eghtesad, M., Daneshmand, F., \& Vahdati, N. (2009). Hybrid sliding mode control of semi-active suspension systems. Smart Materials and Structures, 18(12), 125027.

41. Yokoyama, M., Hedrick, J. K., \& Toyama, S. (2001, June). A model following sliding mode controller for semi-active suspension systems with MR dampers. In Proceedings of the 2001 American Control Conference.(Cat. No. 01CH37148) (Vol. 4, pp. 2652-2657). IEEE.

42. Singla, U. L., \& Singh, S. P. (2004). Semi-active control of automotive vehicle suspension system using magnetorheological damper-a review.

43. Perruquetti, W., \& Barbot, J. P. (2002). Sliding mode control in engineering. CRC press.

44. Zhang, H., Wang, E., Zhang, N., Min, F., Subash, R., \& Su, C. (2015). 
Semi-active sliding mode control of vehicle suspension with magneto-rheological damper. Chinese Journal of Mechanical Engineering, 28(1), 63-75.

45. J. Liu and X. Wang, Advanced sliding mode control for mechanical systems: design, analysis and MATLAB simulation: Springer Science \& Business Media, 2012.

46. L. A. Zadeh, "Fuzzy sets," Information and control, vol. 8, pp. 338353, 1965.

47. Mamdani, E. H. (1977). Application of fuzzy logic to approximate reasoning using linguistic synthesis. IEEE transactions on computers, 26(12), 1182-1191.

48. Rasal, S., Jaganmohan, J., Agashe, S., \& Wani, K. P. (2016). Implementation of fuzzy logic control in semiactive suspension for a vehicle using MATLAB SIMULINK (No. 2016-280035). SAE Technical Paper.

49. Speyer, J. (1979). Computation and transmission requirements for a decentralized linear-quadratic-Gaussian control problem. IEEE Transactions on Automatic Control, 24(2), 266-269.

50. ElMadany, M. M., \& Abduljabbar, Z. S. (1999). Linear quadratic Gaussian control of a quarter-car suspension. Vehicle System Dynamics, 32(6), 479-497. 